\title{
GLUCEMIA Y LIPEMIA EN ESCOLARES CON OBESIDAD EN EL DISTRITO METROPOLITANO DE QUITO, ECUADOR
}

\section{GLYCEMIA AND LIPEMIA IN OBESE SCHOOLCHILDREN IN THE METROPOLITAN DISTRICT OF QUITO, ECUADOR}

\section{TITULO CORTO: GLUCEMIA Y LIPEMIA EN ESCOLARES CON OBESIDAD}

\author{
Jacqueline Cevallos-Salazar ${ }^{1,2}$, Oscar Flores-Carrera ${ }^{2,3}$, Pablo Lozano-Ruiz ${ }^{2,4}$, \\ Alexandro Cruz-Mariño ${ }^{2,5,6}$ Miguel Martín-Mateo ${ }^{2,7,8}$ y Natalia Romero-Sandoval ${ }^{1,2,8}$
}

Recibido en Noviembre 12 de 2014

Aceptado en Enero 29 de 2015

\section{RESUMEN}

El exceso de peso es un problema epidémico en el mundo y está presente desde edades tempranas.El objetivo fuer analizar la variabilidad del exceso de peso, glucosa, triglicéridos, colesterol total, lipoproteínas de baja y alta densidad en estudiantes con obesidad. 276 escolares de 9 a 16 años de unidades educativas municipales del Distrito Metropolitano de Quito, cohorte 2010 - 2011, a quienes se les realizó la medición en sangre, tras ayuno de ocho horas. La categorización de riesgo se basó en la American Diabetes Association y el National Cholesterol Education Program. El exceso de peso fue calculado en kilogramos a partir del referente de OMS para edad y sexo. Los adolescentes medios presentaron mayor exceso de peso a diferencia de los adolescentes tempranos y los niños ( $p<0,001$ ). Se encontró alteración de glucemia en el 1,4\%, triglicéridos 24,7\%, colesterol total 8,4\%, lipoproteínas de alta densidad y baja densidad, $31,6 \%$ y 6,2\%, respectivamente. Se encontró correlación positiva y significativa de triglicéridos con un aumento de LDL $(\mathrm{r}=14)$ y de colesterol total $(\mathrm{r}=0,27)$, y con disminución de la fracción HDL $(r=-0,29)$. Las variaciones de biomarcadores descritos deben propiciar el fortalecimiento de las políticas públicas para prevención de la obesidad.

Palabras clave: Obesidad; niño; adolescente; glucosa; colesterol; triglicéridos.(fuente DeCS)

1 MD. Especialista en Medicina Familiar y Comunitaria, Postgrado de Medicina Familiar y Comunitaria, Instituto Superior de Postgrado, Universidad Central del Ecuador, Ecuador. Correo: maxipay76@hotmail.com

2 Grups de Reserca de America i Africa Llatines - GRAAL, Quito, Ecuador.

3 MD. Médico magister en Salud ocupacional, Postgrado de Medicina Familiar y Comunitaria, Instituto Superior de Postgrado, Universidad Central del Ecuador, Ecuador. Correo: oscarflorescarrera@gmail.com

4 MD. Especialista en Medicina Familiar y Comunitaria, Director del Hospital Básico San Gabriel (e), Ministerio de Salud Pública del Ecuador, Ecuador Correo: drpablolozano@yahoo.com

5 MD. Médico postgradista en Medicina Familiar y Comunitaria, Universidad Central del Ecuador.

6 Unidad Metropolitana de Salud Norte, Municipio del Distrito Metropolitano de Quito, Quito, Ecuador. Correo: alexandrocruz1981@hotmail.com

7 PhD, Departamento de Bioestadística, Facultad de Medicina, Universidad Autónoma de Barcelona, Barcelona, España. Correo: miquel.martin@uab.cat

8 PhD, Carrera de Medicina, Facultad de Ciencias Médicas, de la Salud y de la Vida, Universidad Internacional del Ecuador, Quito, Ecuador. Correo: nromero@

internacional.edu.ec 


\section{ABSTRACT}

Obesity is an epidemic problem in the world and is present from an early age. The objective was to analyze the variability of excess weight, glucose, triglycerides, total cholesterol, LDL and HDL in obese students. 276 school children, 9 to 16 years of municipal educational units of the Metropolitan District of Quito, cohort 2010-2011, whom blood measurement was performed after fasting for eight hours. The risk categorization was based on the American Diabetes Association and the National Cholesterol Education Program. Excess weight was calculated in kilograms, from the WHO reference for age and sex. The average adolescents showed greater excess weight unlike early adolescents and children ( $\mathrm{p}<0.001)$. Glucose risk in $1.4 \%$, triglycerides $24.7 \%$, total cholesterol $8.4 \%$, HDL and LDL $31.6 \%$ and $6.2 \%$ respectively was found. Significant positive correlation was found with triglycerides increased LDL $(r=14)$ and total cholesterol $(r=0.27)$, and decreased HDL $(r=-0.29)$ fraction. Changes in biomarkers described should facilitate the strengthening of public policies for obesity prevention.

Keywords: Obesity; children; adolescents; glucose; cholesterol; triglycerides.(Fuente DeCS)

\section{INTRODUCCIÓN}

$\mathrm{L}$ a Organización Mundial de la Salud (OMS) define al ssobrepeso y obesidad como un depósito excesivo y anómalo de grasa con afectación a la salud y declaró a la obesidad como la epidemia del siglo XXI por su alta prevalencia ${ }^{1}$. En niñas, niños y adolescentes el problema del exceso de peso es cada vez más manifiesto y está presente en todos los estratos; para el año 2005 el porcentaje de sobrepeso y obesidad en niños de países de altos ingresos fue del 10,6\%, mientras que en los países de bajos ingresos fue de $5,2 \%$, prevalencias que se han duplicado y que sobrepasan el $20 \%$ de acuerdo con recientes publicaciones ${ }^{2,3}$.

La obesidad constituye un factor de riesgo para los más jóvenes pues el exceso de peso por sí solo es un elemento asociado al debut de enfermedades crónicas no transmisibles (ECNT) como: diabetes, hipertensión arterial, patologías coronarias, pulmonares, así como del aparato locomotor y algunos tipos de cáncer ${ }^{4,5}$. La aparición de exceso de peso en la infancia, junto a la adopción de hábitos sedentarios y prácticas alimentarias no saludables, tiende a perpetuarse en la etapa adulta; por lo que, niñas, niños y adolescentes obesos tienen mayor riesgo de convertirse en adultos obesos con la carga de enfermedad referida ${ }^{6}$.

En niñas, niños y adolescentes con obesidad los valores elevados del índice de masa corporal (IMC) se asocian con la acumulación excesiva de tejido adiposo ${ }^{7}$; sin embargo, hay limitados estudios sobre la variabilidad de la glucosa y los lípidos en función del exceso de peso. En Ecuador, en el año 2012 el estudio "Quito municipal schools" cohort study: Baseline results" publicó como resultados principales la prevalencia de sobrepeso y de obesidad en el orden del $18,7 \%$ y $7,9 \%$ respectivamente, lo que permite concluir que uno de cada cuatro estudiantes presentan exceso de peso ${ }^{8}$.

Entidades de control del sistema escolar al que pertenecen los estudiantes vieron la necesidad de extender la valoración clínica con el estudio de marcadores bioquímicos como glucosa, colesterol total, lipoproteínas de alta densidad (HDL), liproteínas de baja densidad (LDL) y triglicéridos y analizar el exceso de peso, con la meta de detectar posibles modificaciones subclínicas, su manejo y seguimiento. El objetivo del presente estudio fue analizar el comportamiento estadístico de los mencionados biomarcadores en niños y adolescentes con obesidad.

\section{MATERIALES Y MÉTODOS}

Los participantes fueron estudiantes de sexto a décimo nivel, de 16 Unidades Educativas Municipales del Distrito Metropolitano de Quito (DMQ), cohorte 2010 - 2011, en edades comprendidas entre los 9 y 16 años, con criterios de obesidad para edad y sexo de acuerdo con los parámetros de la OMS (IMC P 97), en quienes se realizó 
la medición en sangre de glucosa y lípidos (colesterol, triglicéridos, lipoproteínas de baja densidad- LDL y de alta densidad - HDL), tras ayuno de ocho horas.

Las muestras de sangre fueron procesadas en el laboratorio de la Unidad Metropolitana de Salud Centro acreditado por la Secretaría de Salud del DMQ; el equipo utilizado es de tipo automático marca Biolis 24i Premium, el método empleado fue enzimático colorimétrico para glucosa, colesterol total, HDL y triglicéridos, el valor de LDL fue calculado con la fórmula de Friedewald, exceptuando valores de triglicéridos mayores a $400 \mathrm{mg} /$ dl. El análisis excluye aquellos sujetos con enfermedades crónicas, gestación, incumplimiento del ayuno y no deseo de participación.

La categorización de los biomarcadores se construyó bajo criterios internacionales de instituciones referentes en el tema como: American Diabetes Association (ADA), National Heart, Lung, and Blood Institute (NHLBI) y National Cholesterol Education Program (NCEP), para niños y adolescentes.

De acuerdo con las recomendaciones de la ADA, un valor de glucemia en ayunas menor a $100 \mathrm{mg} / \mathrm{dl}$ es considerado como normal, valores entre 100 a $126 \mathrm{mg} / \mathrm{dl}$ es denominado como glucosa alterada en ayunas y sobre $126 \mathrm{mg} / \mathrm{dl}$ se considera criterio de diabetes ${ }^{9}$.

Para la categorización de los diferentes lípidos, se asumieron los criterios emitidos por NCEP y publicados en el 2011 por NHLBI. Los puntos de corte, a partir de los cuales se considera de riesgo, para colesterol total, LDL y triglicéridos son aquellos que sobrepasan el percentil 95 para la edad o supera el valor de Colesterol total de $200 \mathrm{mg} / \mathrm{dl}$, LDL mayor o igual a $130 \mathrm{mg} / \mathrm{dl}$ y Triglicéridos mayor o igual a $100 \mathrm{mg} / \mathrm{dl}$ a los 9 años y mayor o igual a $130 \mathrm{mg} / \mathrm{dl}$ a partir de los 10 años. Para HDL el riesgo se considera cuando el valor está por debajo del percentil 10 para la edad o menor a $40 \mathrm{mg} / \mathrm{dl}$. Para el estudio se tomó en cuenta solo los valores catalogados como normales y de riesgo, y, se consideró que la alteración de cualquiera de los parámetros (en forma aislada o en combinación con otros) fue suficiente para definir la presencia de dislipemia ${ }^{10,11}$.

Las variables continuas como edad, exceso de peso (cantidad de kilogramos por sobre el valor referencial para edad y sexo según OMS), glucemia y lípidos se describen a través de medidas de tendencia central, dispersión y posición; las variables categóricas como sexo y, una vez recategorizadas la edad, glucemia y lípidos, se detallan por frecuencias. El grado de relación entre las variables continuas fue analizado mediante el Coeficiente de Correlación de Pearson con prueba de significación bilateral $\mathrm{p}<0.05$. La comparación de medianas de exceso de peso (Kg) entre los grupos de edad se realizó con pruebas no paramétricas (Kruskal-Wallis). Debido a lo robusto del análisis de la varianza se usó para analizar las diferencias entre las medias en los grupos de edad a posteriori con el test Bonferroni. Se utilizó el paquete estadístico SPSS v. 20.

\section{Declaración de aspectos éticos}

La fuente de datos en que se basa el estudio sigue los preceptos de la Declaración de Helsinki de la Asociación Médica Mundial y garantiza la confidencialidad de las personas participantes. La aprobación académica del estudio fue obtenida por la Secretaría de Salud del Municipio del Distrito Metropolitano de Quito y por la Dirección del Instituto Superior de Postgrado de la Facultad de Ciencias Médicas de la Universidad Central del Ecuador. La aprobación final del protocolo fue obtenida por el Comité de Padres de Familia, el Consejo de los Estudiantes y el Consejo de Profesores de cada una de las unidades educativas. El consentimiento informado fue entregado a los padres de los participantes al inicio del estudio. La autorización para la participación fue debidamente firmada por los padres o representantes y además se contó con la aprobación de los estudiantes, ningún estudiante rechazó participar. Para el manejo de la información se mantuvo el cifrado de la identificación de cada estudiante lo que asegura el anonimato y la reserva de los datos.

\section{RESULTADOS}

La población de estudio estuvo conformada por 276 estudiantes con parámetros de obesidad, de los cuales $68,8 \%$ (190) fueron de sexo masculino, la media de edad fue 11,5 años (DT 1,5). Para efectos del estudio la edad de los participantes se recategorizó en tres grupos etarios, de acuerdo con la clasificación de la $\mathrm{OMS}^{12,13}$, nueve años o etapa de niñez que representó el 6,5\% en el estudio, el grupo de 10 a 13 años o adolescencia temprana $(85,2 \%)$ y de 14 a 16 años o adolescencia media (8,3\%); no se encontraron participantes mayores de 17 años para considerar a la adolescencia tardía.

El exceso de peso estuvo comprendido entre 4,3 y 39,9 Kg, promedio 11,6 (DT 5,3), mediana 10,6; el 10\% de estudiantes presentaron 17,5 kg o más. Los adolescentes 
entre 14 y 16 años o en etapa de adolescencia media mostraron una mediana de exceso de peso de $13,1 \mathrm{~kg}$, a diferencia de los participantes en adolescencia temprana y niñez que presentaron 10,4 y $8,1 \mathrm{~kg}$, respectivamente $(\mathrm{p}<0,001)$. El análisis de la varianza mostró que los adolescentes medios se diferencian de los adolescentes tempranos y los niños y no entre estos dos últimos $(\mathrm{p}=<0,001$; grados de libertad $=2)$. El análisis de la tendencia lineal entre las dos variables tiene un $r=0,43$ $(\mathrm{p}=<0,001)$, en las niñas se encontró un valor $\mathrm{r}=0,50$ $y$ en los niños $0,38(p<0,001)$.
La media de los valores de glucemia fue $82,4 \mathrm{mg} / \mathrm{dl}$ (DT 7,4), el valor mínimo $62,0 \mathrm{mg} / \mathrm{dl}$ y máximo $108,0 \mathrm{mg} / \mathrm{dl}$. El colesterol total presentó una media de 155,8 mg/dl (DT 30.2 ), alrededor del $5 \%$ de los estudiantes mostraron el colesterol mayor o igual a $206 \mathrm{mg} / \mathrm{dl}$. Con respecto a las lipoproteínas, la media de HDL fue 45,7 (DT 10,2), el 10\% de los participantes presentaron valores de HDL menores a $35 \mathrm{mg} / \mathrm{dl}$ y el $5 \%$ valores mayores a $130 \mathrm{mg} / \mathrm{dl}$ de LDL, de la cual el promedio fue $91.8 \mathrm{mg} / \mathrm{dl}$ (DT 24,9). El valor promedio de los triglicéridos fue $100 \mathrm{mg} / \mathrm{dl}(\mathrm{DT} 51,9)$, en el percentil 95 se encontró el valor 198,4 mg/dl (Tabla 1).

Tabla 1. Descripción estadística de los parámetros lipídicos en los estudiantes con obesidad cohorte 2010-2011

\begin{tabular}{|c|c|c|c|c|c|c|c|c|c|c|c|c|c|}
\hline \multirow{3}{*}{ Edad } & \multirow{3}{*}{$\mathbf{n}$} & \multicolumn{3}{|c|}{ Colesterol total } & \multicolumn{3}{c|}{ Colesterol LDL } & \multicolumn{3}{c|}{ Colesterol HDL } & \multicolumn{4}{c|}{ Triglicéridos } \\
\cline { 3 - 15 } & & \multicolumn{3}{|c|}{ Percentiles } & \multicolumn{3}{c|}{ Percentiles } & \multicolumn{3}{c|}{ Percentiles } & \multicolumn{3}{c|}{ Percentiles } \\
\cline { 3 - 16 } & & $\mathbf{5 0}$ & $\mathbf{7 5}$ & $\mathbf{9 0}$ & $\mathbf{5 0}$ & $\mathbf{7 5}$ & $\mathbf{9 0}$ & $\mathbf{5 0}$ & $\mathbf{7 5}$ & $\mathbf{9 0}$ & $\mathbf{5 0}$ & $\mathbf{7 5}$ & $\mathbf{9 0}$ \\
\hline 9 & 18 & 166.0 & 173.0 & 218.0 & 96.6 & 104.9 & 148.0 & 44.5 & 52.8 & 61.3 & 98.5 & 129.3 & 190.0 \\
\hline 10 & 75 & 155.0 & 165.0 & 191.2 & 88.4 & 106.1 & 119.2 & 45.0 & 54.0 & 62.7 & 93.0 & 125.0 & 188.8 \\
\hline 11 & 45 & 156.0 & 179.5 & 198.4 & 93.6 & 108.7 & 126.5 & 45.0 & 52.7 & 58.7 & 84.0 & 115.5 & 178.6 \\
\hline 12 & 54 & 145.5 & 166.3 & 196.0 & 83.9 & 100.5 & 119.5 & 40.5 & 47.2 & 56.8 & 87.5 & 131.5 & 177.5 \\
\hline 13 & 61 & 156.0 & 180.0 & 200.0 & 91.0 & 114.3 & 134.6 & 44.0 & 50.9 & 58.6 & 89.0 & 140.0 & 175.6 \\
\hline 14 & 19 & 150.0 & 171.0 & 205.0 & 90.2 & 105.0 & 129.7 & 42.6 & 55.0 & 60.7 & 83.0 & 107.0 & 164.0 \\
\hline 15 & 1 & 200.0 & 200.0 & 200.0 & 100.6 & 100.6 & 100.6 & 36.0 & 36.0 & 36.0 & 67.0 & 67.0 & 67.0 \\
\hline 16 & 2 & 140.0 & - & - & 86.6 & - & - & 41.0 & - & - & 62.0 & - & - \\
\hline
\end{tabular}

Los biomarcadores estudiados en todos los participantes, una vez categorizados como factor de riesgo, según se describió en la sección de Materiales y Métodos, evidenciaron los siguientes porcentajes: hiperglucemia $1,4 \%$, hipertrigliceridemia $24,7 \%$, hipercolesterolemia total 8,4\%, lipoproteínas de alta densidad de riesgo 31,6\% y lipoproteína de baja densidad de riesgo 6,2\%. Un 50,7\% de los estudiantes tuvieron alguna dislipemia, el 14,5\% tuvo dos tipos de dislipemia y el 2,2\% tres tipos.
Las correlaciones bivariada entre las variables exceso de peso y biomarcadores se encuentran en la tabla 2. Las relaciones significativas se encuentran entre triglicéridos, colesterol total, HDL y LDL y se expresa el $r^{2}$ en el caso de significancia. El aumento de triglicéridos se asocia con un aumento de LDL $(\mathrm{r}=0.14)$ y de colesterol total $(\mathrm{r}=0.27)$, a esto se añade que disminuye HDL $(r=-0.29)$. La asociación entre colesterol y HDL $(r=0,27)$, LDL $(r=0.84)$ es directamente proporcional. No existe asociación entre las dos fracciones del colesterol. 
Tabla 2. Correlación $\mathrm{r}\left(\mathrm{R}^{2}\right)$ : exceso de peso y biomarcadores en los estudiantes con obesidad de las Unidades Educativas Municipales del Distrito Metropolitano de Quito, cohorte 2010-2011

\begin{tabular}{|c|c|c|c|c|c|c|c|}
\hline & & $\begin{array}{l}\text { Exceso de } \\
\text { peso }(\mathrm{Kg})\end{array}$ & $\begin{array}{l}\text { Glucosa } \\
\text { (mg/dl) }\end{array}$ & $\begin{array}{c}\text { Colesterol } \\
\text { total }(\mathrm{mg} / \mathrm{dl})\end{array}$ & $\begin{array}{l}\mathrm{HDL}^{\mathrm{a}} \\
(\mathrm{mg} / \mathrm{dl})\end{array}$ & $\begin{array}{l}\mathrm{LDL}^{\mathrm{b}} \\
(\mathrm{mg} / \mathrm{dl})\end{array}$ & $\begin{array}{c}\text { Triglicéridos } \\
\text { (mg/dl) }\end{array}$ \\
\hline \multirow{3}{*}{ Exceso de peso (Kg) } & Correlación $^{c}$ & 1 & 019 & 033 &,- 053 & ,026 &,- 024 \\
\hline & Valor $\mathrm{p}^{\mathrm{d}}$ & & 757 &, 582 & ,383 & 664 & ,697 \\
\hline & $\mathrm{n}$ & 276 & 276 & 275 & 275 & 275 & 275 \\
\hline \multirow{3}{*}{ Glucosa $(m g / d l)$} & Correlación & 019 & 1 & ,114 & 081 & ,071 & ,079 \\
\hline & Valor $\mathrm{p}$ & ,757 & & 059 & 180 & 239 & , 194 \\
\hline & $\mathrm{n}$ & 276 & 276 & 275 & 275 & 275 & 275 \\
\hline \multirow{4}{*}{ Colesterol total $(\mathrm{mg} / \mathrm{dl})$} & Correlación & 033 & ,114 & 1 & $271^{* *}$ & $842^{* *}$ & $268^{* *}$ \\
\hline & Valor $\mathrm{p}$ &, 582 & 059 & &, 000 & ,000 &, 000 \\
\hline & $\mathrm{R}^{2 \mathrm{e}}$ & & & & $7,3 \%$ & $70,9 \%$ & $7,2 \%$ \\
\hline & $\mathrm{n}$ & 275 & 275 & 275 & 275 & 275 & 275 \\
\hline \multirow{4}{*}{$\mathrm{HDL}(m g / d l)$} & Correlación &,- 053 & 081 & $271^{* *}$ & 1 & ,113 &,$- 289^{* *}$ \\
\hline & Valor $\mathrm{p}$ & ,383 &, 180 & 000 & & 061 &, 000 \\
\hline & $\mathrm{R}^{2}$ & & & & & & $7,4 \%$ \\
\hline & $\mathrm{n}$ & 275 & 275 & 275 & 275 & 275 & 275 \\
\hline \multirow{4}{*}{ LDL (mg/dl) } & Correlación &, 026 & 071 & $842^{* *}$ & ,113 & 1 &, $143^{*}$ \\
\hline & Valor $\mathrm{p}$ & ,664 & ,239 & ,000 & 061 & & ,018 \\
\hline & $\mathrm{R}^{2}$ & & & & & & $2,0 \%$ \\
\hline & $\mathrm{n}$ & 275 & 275 & 275 & 275 & 275 & 275 \\
\hline \multirow{4}{*}{ Triglicéridos (mg/dl) } & Correlación &,- 024 & ,079 & $268^{* *}$ &,$- 289^{* *}$ & $143^{*}$ & 1 \\
\hline & Valor $\mathrm{p}$ & ,697 & , 194 &, 000 &, 000 &, 018 & \\
\hline & $\mathrm{R}^{2}$ & & & $7,2 \%$ & $7,4 \%$ & $2,0 \%$ & \\
\hline & $\mathrm{n}$ & 275 & 275 & 275 & 275 & 275 & 275 \\
\hline
\end{tabular}

${ }^{a}$ HDL: Lipoproteína de alta densidad

${ }^{b}$ LDL: Lipoproteína de baja densidad

c Correlación: Valor calculado del estadístico de Correlación de Pearson (r)

${ }^{d}$ Valor p: Valor de significancia

${ }^{e} R^{2}$ coeficiente de determinación

**La correlación es significativa al nivel 0,01 (bilateral)

*La correlación es significante al nivel 0,05 (bilateral)

\section{DISCUSIÓN}

En el grupo de estudio el exceso de peso, catalogado como el excedente de masa corporal expresada en kilogramos, es considerable tomando en cuenta que esta categoría se construye para cada estudiante basado en percentiles según la talla, edad y sexo; la obesidad en la población infantil y juvenil tiende a persistir en la edad adulta. En este estudio se encontró que el exceso de peso registrado en los adolescentes entre 14 y 16 años se diferenció significativamente de aquel encontrado en los niños y adolescentes tempranos. Estudios sobre seguimiento 
(tracking) de parámetros antropométricos describen esta asociación, el estudio de cohorte Bogalusa, tras 17,6 años de seguimiento, publicó que un IMC alto en tempranas edades se asocia directamente con el exceso de adiposidad en la edad adulta ${ }^{14}$.

La relación entre la edad y el exceso de peso en este grupo de participantes muestra una clara tendencia directamente proporcional, con un coeficiente de determinación $\mathrm{r}^{2}=0,20$, lo cual indica que, en ausencia de la consideración de otros factores, la edad por si misma ya explica un $20 \%$ de la variabilidad del exceso de peso. Este estudio constata el efecto de que con la edad se acumula más exceso de peso, por un lado; y por otro que este efecto es mayor en las niñas $\left(r^{2}=0,25\right)$. En las niñas el cambio de edad explica el $25 \%$ de la variación del exceso de peso, efecto que en los niños es del 15\% $\left(r^{2}=0,15\right)$. Estos datos aportan al análisis realizado en todo la población participante en la cohorte indicada, en el que se encontró que en los hombres con exceso de peso la aportación de la grasa para explicar el peso fue menor que la talla y en las mujeres la aportación de la grasa corporal y la talla fue igual en todas las edades ${ }^{15}$.

En el grupo estudiado se encontró un 1,4\% con glucemia alterada en ayunas, otros trabajos señalan porcentajes más elevados. Sinha et al., describió la prevalencia de $22 \%$ de alteración de glucemia en 114 adolescentes con criterio de obesidad; los participantes fueron sometidos a un test de tolerancia de glucosa, insulinemia y péptido $C$, estándares más exhaustivos para el diagnóstico ${ }^{16}$.

En el estudio ORANGE 2, en un grupo de 15 a 19 niños y adolescentes, entre seis y 19 años, la prevalencia de intolerancia a la glucosa encontrada fue $3,7 \%$, con un incremento del $12,7 \%$ en niños con obesidad abdominal ${ }^{17}$.

Con respecto a los lípidos, prácticamente uno de cada cuatro estudiantes presentó hipertrigliceridemia $(24,7 \%)$, uno de cada tres valores de riesgo para LDL colesterol $(31,6 \%)$ y uno de cada dos escolares presentaron algún tipo de dislipemia $(50,7 \%)$. La prevalencia de dislipemia en niños y adolescentes sigue siendo un tema sensible ya que varían los parámetros considerados.

En el estudio ERICA la prevalencia general de dislipemia en estudiantes argentinos fue de $15,9 \%$, basado en la definición de la Academia Americana de Pediatría, el $41 \%$ presentaron sobrepeso y obesidad ${ }^{18}$. El estudio de Arjona Ortegón en Costa Rica publicó como prevalencia de hiperglicemia $3,1 \%$, hipertrigliceridemia $12 \%$, HDL bajo $9,9 \%$ y LDL elevado $13,3 \%$ basado en la
Asociación Americana del Corazón y la Asociación Americana de Diabetes ${ }^{19}$. En Chile, Barja et al encontró en 3325 estudiantes, con edades entre 10 y 14 años, los promedios de lípidos: colesterol total 159,2 mg/dl, HDL $51,9 \mathrm{mg} / \mathrm{dl}$, LDL $89,0 \mathrm{mg} / \mathrm{dl}$ y triglicéridos $93,2 \mathrm{mg} / \mathrm{dl}$, el exceso de peso se asoció con el aumento significativo de triglicéridos, junto a la disminución del colesterol HDL ${ }^{20}$.

El incremento en los triglicéridos explica el aumento del colesterol en un 7,5\%, $\left(\mathrm{r}^{2}=0.075\right)$ de LDL en un 2,0\% $\left(r^{2}=0.02\right)$ y la disminución de HDL en un $8,4 \%\left(r^{2}=0,84\right)$ en ausencia de otros factores. La asociación entre colesterol y HDL y LDL fue directamente proporcional, el incremento de colesterol explicó el 70,9\% del incremento de la fracción LDL $\left(r^{2}=0,709\right)$. Estos hallazgos dan una amplia visión del comportamiento de los lípidos en adolescentes con obesidad. La alteración en las concentraciones "normales" de lípidos (dislipemia) en la adolescencia ha mostrado una tendencia al incremento rápido lo que podría causar la epidemia de enfermedades cardiovasculares. La edad junto al sexo constituyen los elementos cardinales en la adolescencia que obligan a considerarlos para la clasificación de dislipemia por su variabilidad fisiológica en esta etapa de la vida ${ }^{21}$. La expresión clínica de la dislipemia en la adolescencia es casi inexistente, mientras que al llegar a la adultez son bien conocidas las distintas formas de ateroesclerosis ${ }^{22}$. Los hallazgos del comportamiento de los lípidos expresan el proceso de aumento de la lipólisis y una disminución de la capacidad de los tejidos para acumular triglicéridos con su elevación en sangre, al igual que sus transportadores LDL y VLDL. ${ }^{23}$. Estos resultados aportan con información complementaria a la evaluación clínica ${ }^{8}$, con el fin de implementar intervenciones para la prevención primaria y secundaria.

Los biomarcadores en niños y adolescentes tienen un comportamiento variable al ser un período demandante de energía para el crecimiento, desarrollo y generación de hormonas ${ }^{24}$, el análisis no encontró asociación significativa entre el exceso de peso y la variación de los niveles de glucemia o lípidos, pero muestra la correlación entre los diferentes marcadores lipídicos; la variabilidad del colesterol total y triglicéridos se modifica directamente en función del aumento del LDL y disminución del HDL, alteraciones consideradas como factor de riesgo cardiovascular, presentes en jóvenes como modificación mediata de la obesidad ${ }^{25}$.

En general, frente a las diferentes prevalencias en los diversos estudios sobre el comportamiento de biomarcadores en niños y adolescentes, varias 
publicaciones a largo plazo han demostrado el inicio de las ECNT durante la adolescencia con cambios puntuales en el metabolismo de los lípidos como en la resistencia a la insulina, por lo que la prevención temprana cobra importancia ${ }^{26}$.

La pubertad y la adolescencia son etapas del desarrollo determinadas por los cambios físicos, metabólicos y hormonales, además de la reestructuración de la esfera afectiva, lo que edifica los pilares del pensamiento y comportamiento del adulto. La adolescencia es el período de tiempo donde se forjan los hábitos, costumbres y rutinas con gran influencia de numerosas variables del entorno familiar, escolar y social.

Conocer la caracterización bioquímica de estos parámetros ayudará a definir riesgo en los más jóvenes, de ello el presente análisis deja el planteamiento para nuevas intervenciones con grupos comparativos.

\section{DECLARACIÓN SOBRE CONFLICTO DE INTERESES}

Por la presente los autores declaramos que el artículo "Glucemia y Lipemia en escolares con obesidad en el Distrito Metropolitano de Quito", es independiente con respecto a las instituciones de apoyo, y, que durante la ejecución del trabajo o la redacción del manuscrito, no han incidido intereses o valores distintos a los que usualmente tiene la investigación.

La Secretaría de Salud del Municipio del Distrito Metropolitano de Quito, a través del laboratorio de la Unidad Metropolitana de Salud Centro, facilitó la realización del análisis de muestras sanguíneas. El Instituto Superior de Investigación y Postgrado de la Facultad de Ciencias Médicas de la Universidad Central del Ecuador y la Facultad de Ciencias Médicas, de la Salud y de la Vida de la Universidad Internacional del Ecuador otorgaron su respaldo institucional para la construcción del presente artículo.

\section{AGRADECIMIENTOS}

Los autores agradecen a la Secretaría Metropolitana de Salud del Distrito Metropolitano de Quito, al equipo de flebotomistas y técnicos de laboratorio de la Unidad Metropolitana de Salud Centro, al grupo de médicos residentes del Postgrado de Medicina Familiar y Comunitaria de la Universidad Central del Ecuador cohorte 2010-2013, 2011-2014. Los autores Cevallos J, y
Cruz A fueron becarios de la Secretaría Metropolitana de Salud del Distrito Metropolitano de Quito.

\section{REFERENCIAS BIBLIOGRÁFICAS}

1. Organización Mundial de la Salud. Obesity and overweight [Internet]. WHO. 2013 [citado 11 de noviembre de 2013]. Recuperado a partir de: http:// www.who.int/mediacentre/factsheets/fs311/en/index. html \#6286

2. De Onis M, Blössner M, Borghi E. Global prevalence and trends of overweight and obesity among preschool children. Am J Clin Nutr. 2010;92(5):1257-64.

3. Schmidt MC, Rokholm B, Sjöberg BC, Schou AC, Geisler AL, Rasmussen M, et al. Trends in Prevalence of Overweight and Obesity in Danish Infants, Children and Adolescents - Are We Still on a Plateau? PLoS ONE. 2013;8 (7):e69860.

4. Rao G. Office-Based Strategies for the Management of Obesity - American Family Physician. Am Fam Physician. junio de 2010;(81):1449-55.

5. Renehan AG, Soerjomataram I, Tyson M, Egger M, Zwahlen M, Coebergh JW, et al. Incident cancer burden attributable to excess body mass index in 30 European countries. Int J Cancer J Int Cancer. 1 de febrero de 2010;126 (3):692-702.

6. Freedman DS, Mei Z, Srinivasan SR, Berenson GS, Dietz WH. Cardiovascular Risk Factors and Excess Adiposity Among Overweight Children and Adolescents: The Bogalusa Heart Study. J Pediatr. enero de 2007;150(1):1217.e2.

7. Romero VE, Vásquez GE, Álvarez RY, Fonseca RS, Casillas TE, Troyo SR. Circunferencia de cintura y su asociación con factores de riesgo cardiovascular en niños y adolescentes con obesidad. Bol. Med. Hosp. Infant. Mex. [revista en la Internet]. 2013 [citado 2014 Nov 10] ; 70(5): 358-363. Disponible en: http://www.scielo. org. $\mathrm{mx} /$ scielo.php script $=$ sci_arttext\&pid $=$ S1665$11462013000500004 \& \operatorname{lng}=$ es.

8. Romero SN, Ruiz V, Quizanga J, Recalde R, Anchali E, Falconí J, et al. «Quito municipal schools» cohort study: Baseline results. 2012 [citado 4 de octubre de 2013]; Recuperado a partir de: http://www.scirp.org/journal/ PaperDownload.aspx? paperID $=21941$

9. Association $A D$, others. Standards of medical care in diabetes-2013. Diabetes Care. 2013;36(S1):S11.

10. Daniels S, Benuck I, Christaki D. Expert panel on integrated guidelines for cardiovascular health and risk reduction in children and adolescents: full report. 2012.

11. Subcomisión de Epidemiología, Comité de Nutrición C. Consenso sobre factores de riesgo de enfermedad cardiovascular en pediatría. Obesidad. Arch Argent Pediatr. 2005;103(3):262. 
12. Reynoso F. Valores generales en una muestra de adolescentes medios de la ciudad de Santo Domingo. Cienc Soc [Internet]. 2002 [citado 19 de octubre de 2013];25(4). Recuperado a partir de: http://biblioteca. intec.edu.do/bases/banal/texto/CISO20022704-591-607. PDF

13. Aliño SM, López EJ, Navarro FR. Adolescencia: Aspectos generales y atención a la salud. Rev Cuba Med Gen Integral. 2006;22(1).

14. Freedman DS, Khan LK, Serdula MK, Dietz WH, Srinivasan SR, Berenson GS. The Relation of Childhood BMI to Adult Adiposity: The Bogalusa Heart Study. Pediatrics. 1 de enero de 2005;115(1):22-7.

15. Carvajal D, Martin M, Romero-SN. Modelo explicativo del efecto de la talla y grasa corporal en el peso de escolares entre 9 y 17 años de edad. Rev Med Vozandes 2013; 24: 9-18.

16. Sinha R, Fisch G, Teague B, Tamborlane WV, Banyas B, Allen K, et al. Prevalence of impaired glucose tolerance among children and adolescents with marked obesity. N Engl J Med.2002; 346(11):802-10.

17. Ranjani H, Sonya J, Anjana RM, Mohan V. Prevalence of glucose intolerance among children and adolescents in urban South India (ORANGE-2). Diabetes Technol Ther. enero de 2013;15(1):13-9.

18. Abraham W, Blanco G, Coloma G, Cristaldi A, Gutiérrez N, Sureda L, et al. Estudio de los factores de Riesgo Cardiovascular en Adolescentes. ERICA Study of Cardiovascular Risk factors in Adolescents. Rev Fed Arg Cardiol. 2013;42(1):29-34.

19. Arjona $\mathrm{ON}$, Chávez DS, Romero JJ. Prevalencia y factores asociados a las dislipidemias en niños (as) y adolescentes de Costa Rica. Avances en Seguridad Alimentaria y Nutricional. 2006; 27-41

20. Barja S, Barrios X, Arnaiz P, Domínguez A, Villarroel L, Castillo O, et al. Niveles de lípidos sanguíneos en escolares chilenos de 10 a 14 años de edad. Nutr Hosp. 2013;28(3):719-25.

21. Hatami M, Tohidi M, Mohebi R, Khalili D, Azizi F, Hadaegh F. Adolescent lipoprotein classifications according to National Health and Nutrition Examination Survey (NHANES) vs. National Cholesterol Education Program (NCEP) for predicting abnormal lipid levels in adulthood in a Middle East population. Lipids in health and disease. 2012;11(1): 1-7.

22. Wei FZ, Li Liang, Chun LW, Jun FF. Triglyceride and Non-High-Density Lipoprotein Cholesterol as Predictors of Cardiovascular Disease Risk Factors in Chinese Han Children. Indian Pediatrics. 2013; 50; 394-398

23. Gómez AJ, Rodríguez A, Catalán V, Frühbeck G. Papel del tejido adiposo en la inflamación asociada a la obesidad. Rev Española Obesidad. 2008; 6 (5):264-79.

24. Shutt AJ, Maddaleno M. Salud sexual y desarrollo de adolescentes y jóvenes en las Américas: implicaciones en programas y políticas [Internet]. OPS Washington, DC; 2003 [citado 21 de noviembre de 2013]. Disponible en: http://intpsiquiatria.inteliglobemex.com/inp_piloto/ portal/saludxmi/biblioteca/sexualidad/m3_Factores_ desarrollo_saludsexual_adolescentes.pdf

25. Kaufer HM, Toussaint G. Indicadores antropométricos para evaluar sobrepeso y obesidad en pediatría. Boletín Médico Hosp Infant México. 2008; 65(6):502-18.

26. Zimmet P, Alberti G, Kaufman F, Tajima N, Silink M, Arslanian S, et al. The metabolic syndrome in children and adolescents. The Lancet. 2007;369 (9579):2059-61.

Para citar este artículo: Cevallos-Salazar J, Flores-Carrera O, Lozano-Ruiz P, Cruz-Mariño A, Martín-Mateo M, Romero-Sandoval N. Glucemia y Lipemia en escolares con obesidad en el Distrito Metropolitano de Quito, Ecuador. Duazary. 2015 jun; 12 (1): 7 - 14 